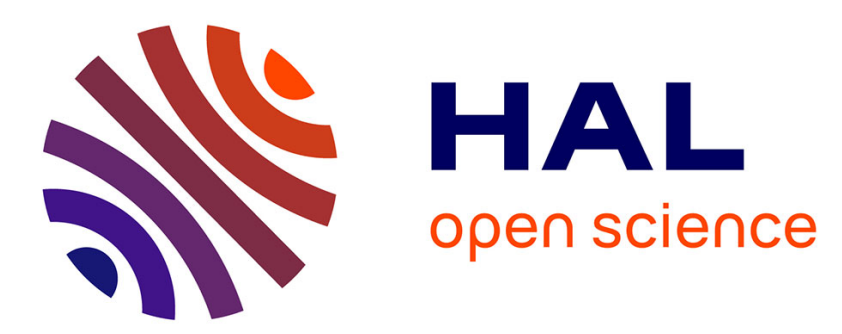

\title{
SMHS1 is involved in oxidative/glycolytic-energy metabolism balance of muscle fibers
}

Didier Pisani, Lilian Leclerc, Gisèle Jarretou, Jean-François Marini, Claude Dechesne

\section{- To cite this version:}

Didier Pisani, Lilian Leclerc, Gisèle Jarretou, Jean-François Marini, Claude Dechesne. SMHS1 is involved in oxidative/glycolytic-energy metabolism balance of muscle fibers. Biochemical and Biophysical Research Communications, 2005, 326 (4), pp.788-793. 10.1016/j.bbrc.2004.11.111 . hal-03219822

\section{HAL Id: hal-03219822 \\ https://hal.science/hal-03219822}

Submitted on 28 Sep 2021

HAL is a multi-disciplinary open access archive for the deposit and dissemination of scientific research documents, whether they are published or not. The documents may come from teaching and research institutions in France or abroad, or from public or private research centers.
L'archive ouverte pluridisciplinaire HAL, est destinée au dépôt et à la diffusion de documents scientifiques de niveau recherche, publiés ou non, émanant des établissements d'enseignement et de recherche français ou étrangers, des laboratoires publics ou privés. 


\section{SMHS1 is involved in oxidative/glycolytic-energy metabolism balance of muscle fibers}

Didier F. Pisani, Lilian Leclerc, Gisèle Jarretou ${ }^{1}$, Jean-François Marini, Claude A. Dechesne ${ }^{2,3}$

Laboratoire de Physiologie Cellulaire et Moléculaire, CNRS UMR 6548, Faculté des Sciences, Parc Valrose, 06108 Nice cedex 2, France.

1 Institut de Pharmacologie Moléculaire et Cellulaire, 660 Route des Lucioles, 06560 Valbonne Sophia-Antipolis, France.

${ }^{2}$ CNRS UMR 6543, Faculté des Sciences, Parc Valrose, 06108 Nice cedex 2, France.

${ }^{3}$ Corresponding author : Institut de Signalisation et Cancer, CNRS UMR 6543, Centre de Biochimie, Faculté des Sciences, Parc Valrose, 06108 Nice cedex 2, France. Tel: +33-4-9207-69-94. Fax: +33-4-92-07-64-04. E-mail address: dechesne@unice.fr 


\begin{abstract}
With the aim of finding important mediators of muscle atrophy, we cloned SMHS1, a novel gene that was found to be upregulated in rat soleus muscle atrophied by restriction of activity. The SMHS1 amino-acid sequence shares $65 \%$ similarity with RTP801 — which is a cellular stress response protein regulated by HIF-1 - but SMHS1 expression was demonstrated to be independent of HIF-1. SMHS1 was found to be mainly expressed in skeletal muscle, and comparisons of its expression in atrophied versus hypertrophied muscles and in oxidative versus glycolytic muscles suggested that SMHS1 contributes to the muscle energy metabolism phenotypes.
\end{abstract}

Key words: skeletal muscle, atrophy, hypertrophy, muscle fiber type, RTP801, HIF-1. 


\section{Introduction}

The well-documented plasticity of skeletal muscle is largely based on the heterogeneity of fiber types which vary in metabolic equipment, contraction speed, and fatigability [1-3]. Briefly, at one end of the classification, slow-twitch oxidative fibers (type I) have a slow contraction time and are fatigue resistant — they are responsible for both maintaining posture and carrying out slow repetitive movements. Then, we found fast-twitch oxidative fibers (type IIa) with a rapid contraction time associated to fatigue resistance. The other end of the classification includes fast-twitch glycolytic fibers (types IIx and IIb), which have a fast contraction time and low fatigue resistance. They are adapted for high-power output. In addition of these principal fibers, it exists several intermediary and transitory fibers (types I/IIa, IIa/IIx and IIx/IIb). Chronic skeletal muscle activity variations cause changes in muscle mass and metabolism, which are associated with shifts in fiber types [4-6]. These well established changes ultimately modify muscle performance, e.g. oxidative slow- to glycolytic fast-twitch transitions in the case of atrophy of slow-twitch muscles (soleus muscle). Many cellular components are involved in this switch, including myofibril protein isoforms, oxidative enzymes, glycolytic enzymes, calcium homeostasis players, transcription factors, and signaling molecules [7-10]. Several previous studies, including those conducted by our laboratory, focused on large-scale gene profiling or proteomic analysis in experimental rat and mouse models of muscle atrophy induced by hindlimb unweighting or immobilization [1116]. In order to identify genes involved in skeletal muscle adaptive responses to chronic activity modifications, we characterized a novel gene that we previously demonstrated to be two-fold upregulated in rat soleus muscles atrophied by restriction of activity imposed by hindlimb suspension (03A12 clone) [12]. This gene, named SMHS1 (Skeletal Muscle Hindlimb Suspension 1), encodes a protein with 65\% similarity (35\% identity) to the RTP801 
protein [17]. RTP801 is a novel recently identified protein under the control of hypoxiainducible factor 1 (HIF-1). After we submitted the full-length SMHS1 cDNA sequence to GenBank the same cDNA sequence was found through in silico EST contigization and named RTP801-like [17].

In this study, we cloned SMHS1 full-length cDNA and characterized the SMHS1 sequence and expression, especially in different muscle mass and energy metabolism conditions. Our data suggest that SMHS1 is a novel gene potentially important for the oxidative versus glycolytic specificities of muscle fibers. 


\section{Materials and Methods}

Animals and tissues. All procedures were approved by the local Centre National de la Recherche Scientifique ethics committee. Ten-week-old Ico: OF1 (Caw) animals were used for mouse tissue RNA preparations. Atrophied soleus and gastrocnemius muscles were derived from a previous study [12]. Ten week-old female Sprague Dawley rats $(\mathrm{n}=9$ / experimental group) were used for soleus muscle compensatory hypertrophies and glycolytic versus oxidative muscle analysis. For hypertrophy experiments, rats were anesthetized and separated in two groups. The first group was sham-operated for control, and the second group was submitted to synergist gastrocnemius muscles ablations to induce a compensatory hypertrophy of plantaris and soleus muscles by overload. Six weeks later, rats were sacrificed and soleus muscles were taken, weight and immediately frozen in liquid nitrogen.

Cell culture. $\mathrm{C}_{2} \mathrm{C}_{12}$ mouse skeletal muscle cells (ATCC $\mathrm{n}^{\circ} \mathrm{CRL}-1772$ ) were grown in Dulbecco's modified Eagle's medium with $4.5 \mathrm{~g} / \mathrm{L}$ glucose and $10 \%$ fetal calf serum. Myotube $\mathrm{C}_{2} \mathrm{C}_{12}$ differentiation was induced by withdrawing fetal calf serum and adding 10 $\mu \mathrm{g} / \mathrm{ml}$ insulin, $5 \mu \mathrm{g} / \mathrm{ml}$ transferrin and $2 \%$ horse serum.

cDNA library screening and cloning of rat SMHS1 full-length cDNA. An oligo(dT)random primed cDNA library of rat skeletal muscle (Clontech) was screened with the 03A12 cDNA fragment [12]. Approximately $1.210^{6} \lambda \mathrm{gt} 11$ phage clones were transfected into host strain Y1090r- and then transferred onto nylon membranes (Macherey-Nagel) for hybridization, as previously described [12]. Positive clones were plaque-purified and the cDNA phage inserts were PCR-purified using $\lambda$ gt1 1 specific vector amplimers. 
RNA analysis. Multiple rat tissue RNA blots were purchased from Clontech Laboratories Inc. Total RNA was prepared with RNAInstaPure kit (Eurogentec). Northern blot analyses were performed as previously described, with ${ }^{32} \mathrm{P}$-labeled mouse partial SMHS1 or actin cDNA and 18S rRNA probes [12]. Reverse transcription reactions were carried out with M-MLV-RT (Invitrogen) and oligo(dT). Advantage Klen Taq polymerase (Clontech) was used for PCR amplification of partial SMHS1 cDNA with the forward primer 5'GAAACAGAGCCGTTGACCAT-3' and the reverse primer 5'-ATTAGCCACTCATTAGG GAC-3'. The 18S rRNA cDNA fragment was amplified with the forward primer 5'-AGTTG GTGGAGCGATTTGTC-3' and the reverse primer 5'-GGCCTCACTAAACCATCCAA-3'. PCR samples were taken in exponential amplification phase, i.e. 25 cycles for SMHS1 and 20 cycles for 18S rRNA. Agarose electrophoretic bands and Northern blot specific signals were quantified using the PCBas software package.

In situ hybridizations were performed on $12 \mu \mathrm{m}$ serial sections of mouse total embryos, at $75^{\circ} \mathrm{C}$ overnight, with 239 bp anti-sense and 400 bp sense SMHS1 digoxigenin-UTP-labeled riboprobes. Riboprobes were synthesized with the DIG RNA labeling kit (Roche), from SMHS1 cDNA cloned in pGEM-T Easy (Promega) using SP6 and T7 transcription starts after plasmid linearization with NheI. Visualization of digoxigenin (DIG)-nucleic acids with alkaline-phosphatase-conjugated anti-DIG antibody was carried out according to Roche's procedure.

Protein analysis. Western blotting analyses were performed on protein whole extracts of wild-type $\mathrm{C} 2 \mathrm{C} 12$ cells as previously described [18]. Anti-HIF-1 $\alpha$ monoclonal antibody (AbCam, clone hif ${ }^{\alpha 67}$ ) and anti-FLAG monoclonal antibody (Sigma) were used at 1:200 and 1:2000 dilution, respectively. 
Statistical analysis. $t$-test was performed using SigmaStat software in order to compare the differences between two groups of values. Statistical differences were noted $*(p<0.05)$. 


\section{Results}

\section{Molecular cloning and sequence analysis of SMHS1}

We previously cloned the 03A12 novel partial cDNA on the basis of its upregulation in rat soleus muscle atrophied after activity restriction induced by hindlimb suspension [12]. This cDNA was used to screen a rat skeletal muscle cDNA library, and a putative $1.1 \mathrm{~kb}$ fulllength cDNA was cloned and named SMHS1 for "Skeletal Muscle Hindlimb Suspension 1". The nucleotide sequence (GenBank accession no. $\underline{\text { AF327511) }}$ ) contained a 579 bp open reading frame compatible with the size of the $\sim 1.2 \mathrm{~kb}$ SMHS1 mRNA. The mouse ortholog cDNA (GenBank accession no. $\underline{\mathbf{A F 3 2 7 5 1 2}}$ ) was cloned through RT-PCR experiments using rat SMHS1 oligonucleotides as primers and mouse muscle RNAs as templates. The open reading frame accuracy was confirmed by expression in $\mathrm{C} 2 \mathrm{C} 12$ muscle cells (not shown). The highest SMHS1 sequence homologies were found with a few vertebrate proteins, including an unknown zebrafish protein and an hypothetical Xenopus tropicalis protein (Fig. 1). The only meaningful sequence homology concerned the $65 \%$ amino acid residue similarity (35\% identity) with a recently identified protein named RTP801 [17], or REDD1 [19], or dig2 [20]. This protein is involved in cellular stress responses and its RNA transcription is regulated by hypoxia-inducible factor 1 (HIF-1). Although no known functional domains were found within the sequences reported in Fig. 1, it was interesting to note the presence of several residue stretches highly identical in the different proteins, especially in the $\mathrm{C}$ terminal portion, suggesting that these domains have been conserved throughout evolution.

\section{SMHS1 mRNA expression}

Hybridization of rat RNAs with the rat SMHS1 cDNA revealed an abundant $\sim 1.2 \mathrm{~kb}$ mRNA with high expression in skeletal muscle, in addition to expression in heart and testis (Fig. 2A). 
In fact, as previously noted with the initial 03A12 clone, two less abundant and longer mRNAs also hybridized with SMHS1 probes, but were not investigated in the present study [12]. The rat expression profile was roughly confirmed in mouse by RT-PCR performed with rat/mouse SMHS1 specific primers and mouse tissue RNA templates. Expression was mainly detected in mouse skeletal muscle and eye, and lower expression was detected in heart and brain (Fig. 2B). Eye RNA was not tested on the above-mentioned rat Northern blots.

SMHS1 mRNA tissue distribution was further confirmed by in situ hybridizations on sections of 18 day-old mouse embryos (Fig. 2C). This revealed expression in skeletal muscle, heart, and brain similar to that in adult mouse/rat (testis and eye expression was not investigated).

\section{SMHS1 expression is independent of HIF-1 variations}

Because of the sequence similarities between SMHS1 and RTP801, which is an HIF-1 target gene, we wondered whether HIF-1 could also regulate SMHS1 expression. HIF-1 comprises two subunits, HIF-1 $\alpha$ and HIF-1 $\beta$, and HIF- $1 \alpha$ is the oxygen-dependent regulatory subunit. We induced $\mathrm{CoCl}_{2}$ cellular stress, which is known to inhibit proteasome-induced HIF-1 $\alpha$ degradation, thus allowing formation of the active HIF-1 $\alpha /$ HIF-1 $\beta$ complex [21]. Murine muscle $\mathrm{C}_{2} \mathrm{C}_{12}$ cells were differentiated in myotubes and stressed with $200 \mu \mathrm{M} \mathrm{CoCl}_{2}$ treatments. Changes in SMHS1 mRNA expression were assessed by semi-quantitative RTPCR in relation with variations in HIF-1 $\alpha$ protein quantities, followed by Western blot analysis. As expected [22], $\mathrm{CoCl}_{2}$ stress induced HIF-1 $\alpha$ protein accumulation, which increased over the $3 \mathrm{~h}$ treatment (Fig. 3A). However, no significant variations in SMHS1 RNA were noted (Fig. 3B-C), suggesting that the HIF-1 transcription factor does not directly control SMHS1 expression.

\section{SMHS1 expression and muscle fiber oxidative/glycolytic-energy metabolism status}


In soleus muscles, activity restriction induces atrophy associated with the transition from a large majority of oxidative fibers to a mixture of oxidative and glycolytic fibers [11]. Figure 4A shows that a $40 \%$ atrophy was seen in soleus muscles after 2 weeks of activity restriction imposed by hindlimb suspension. SMHS1 was found to be two-fold upregulated in such muscles. This upregulation, which was the starting point of the SMHS1 cloning, may be thus related to the muscle mass variation or/and to the energy metabolism shift. To further clarify this point, SMHS1 mRNA expression was analyzed in 3 other situations by semi-quantitative RT-PCR and Northern blots.

First, we checked SMHS1 mRNA expression in the same rat muscle atrophy model but in the predominantly glycolytic gastrocnemius muscle (Fig. 4B). After 2 weeks of activity restriction, this muscle presented a $25 \%$ atrophy and it has been previously demonstrated that under these experimental conditions the gastrocnemius muscle undergoes no variations in myosin isoform, and consequently no variations in energy metabolism type [23, 24]. Interestingly, no significant variation was found in SMHS1 mRNA expression.

Secondly, approximately $50 \%$ hypertrophy of soleus muscle was obtained as a compensatory effect 6 weeks after gastrocnemius muscle ablation (Fig. 4C). Such soleus muscle hypertrophy does not involve changes in the oxidative/glycolytic fiber ratio $[25,26]$ and no alteration of SMHS1 expression was found in the hypertrophied soleus muscles.

Thirdly, we compared SMHS1 mRNA levels in the predominantly glycolytic quadriceps muscle versus the predominantly oxidative soleus muscle, independently of any muscle mass variations. SMHS1 mRNA expression was found to be three-fold higher in the glycolytic muscle than in the oxidative muscle (Fig. 5). 


\section{Discussion}

We report on cloning of a complete cDNA that we named SMHS1 for "Skeletal Muscle Hindlimb Suspension 1". The tissue origins of sequence-matching EST and cDNA clones as well as our Northern blot and RT-PCR data show that SMHS1 expression is restricted to a few tissues, mainly skeletal muscle, and to a lesser extent heart, testis, eye and brain.

As SMHS1 function is unknown, we assessed it in skeletal muscle. Our first line of investigation was based on sequence homologies with RTP801 (Fig.1), a recently described HIF-1-regulated gene. Since SMHS1 expression is regulated in skeletal muscle, as discussed below, HIF-1 regulation of SMHS1 mRNA was considered. This actually seemed unlikely since the upregulation of HIF-1 $\alpha$ protein - which allows the formation of an active HIF$1 \alpha /$ HIF-1 $\beta$ complex - in the $\mathrm{CoCl}_{2}$ treatment of $\mathrm{C}_{2} \mathrm{C}_{12}$ cells had no impact on the SMHS1 mRNA level (Fig.3). The non-control of SMHS1 transcription is further supported by the fact that we did not find any cis-acting nucleotide sequence binding HIF-1 in the SMHS1 gene. This motif is named the hypoxia-responsive element and its consensus sequence is either 5'(G/C/T)ACGTGC(G/C)-3' or 5'-RCGTG-3' [22]. The SMHS1 8 kb-region upstream from the ATG translation initiation start codon was unsuccessfully screened for the presence of the consensus sequence. These observations strongly suggest that SMHS1 is not an HIF-1 responsive gene like RTP801.

To gain further insights in SMHS1 function, we based our in vivo experiments on the initial finding that SMHS1 expression was altered in soleus muscle atrophy due to hindlimb suspension; this was the basis for SMHS1 cloning [12]. In this model, two modifications are primordial, muscle mass decrease and energy metabolism shift [11-16]. To assess the role of SMHS1 in each of these modifications, we analyzed SMHS1 expression in different in vivo 
situations. The situations associated or dissociated muscle mass variations and energy metabolism variations (Fig. 3 and 4). In total, (i) a significant upregulation of SMHS1 mRNA was found in soleus muscle atrophy. The 2-week hindlimb suspension is characterized by a $40 \%$ decrease of muscle mass, and a large oxidative- to glycolytic-energy transition associated to a variation in fiber type content. The amount of type I/IIa oxidative fibers decreases from 100 to $85 \%$ and the amount of type IIx/IIb glycolytic fibers increases from 0 to $15 \%$, according to the myosin heavy chain isoform contents [11]. (ii) Conversely, no significant SMHS1 mRNA alteration was detected during gastrocnemius muscle atrophy due to a 2 -week hindlimb suspension. This muscle was $25 \%$ atrophied and no variation in energy metabolism / fiber type composition was found in this situation [23, 24]. (iii) Our analysis showed a stable SMHS1 mRNA expression in soleus muscle hypertrophy due to a 6-weeks synergists muscles ablation. Soleus muscle was 50\% hypertrophied and it has been shown that this does not imply any significant energy-metabolism switch $[25,26]$. (iv) SMHS1 mRNA expression was finally checked in the predominantly glycolytic gastrocnemius muscle $(70 \%$ type IIb/x fibers and 30\% type I/IIa fibers) compared to the predominantly oxidative soleus muscle (100\% type I/IIa fibers) $[11,23]$, and found to be three-fold higher in the more glycolytic muscle. These results taken together strongly suggest that an higher SMHS1 mRNA expression is associated with muscle glycolytic energy metabolism; SMHS1 expression is related to oxidative/glycolytic metabolism balance and varies with skeletal muscle energy metabolism transitions, independently of muscle mass variations per se.

Many molecules are involved in the development and in the differences between oxidative and glycolytic muscle fibers, and the plasticity between these fibers according to muscle functional constraints [1, 2, 4-7, 27]. SMHS1 is another good candidate molecule that could be involved in such mechanisms, and this could be further supported by the fact that SMHS1 has sequence homologies with RTP801/REDD1/dig2 [17, 19, 20], which is claimed to be 
involved in oxidative stress and hypoxia response. Indeed, muscle fiber energy metabolism is strongly linked to oxygen homeostasis.

Finally, it seems likely that the novel SMHS1 gene, whose exact function still remains to be elucidated, is not a muscle mass variation marker, nor a cellular stress response gene (unlike RTP801/REDD1/dig2), but is involved in the energy metabolism status within skeletal muscle fibers. It will be interesting to find out what is the exact SMHS1 contribution to the skeletal muscle fiber phenotypes.

Acknowledgments: We are grateful to David Manley for the English revision of the manuscript. D. F. Pisani is a recipient of grants from the Association Française contre les Myopathies and the Fondation pour la Recherche Médicale. 


\section{References}

[1] D. Pette, and R. S. Staron, Cellular and molecular diversities of mammalian skeletal muscle fibers, Rev Physiol Biochem Pharmacol. 116 (1990) 1-76.

[2] S. Schiaffino, and C. Reggiani, Molecular diversity of myofibrillar proteins: gene regulation and functional significance, Physiol Rev. 76 (1996) 371-423.

[3] M. Zhang, K. Koishi, and I. S. McLennan, Skeletal muscle fibre types: detection methods and embryonic determinants, Histol Histopathol. 13 (1998) 201-207.

[4] R. H. Fitts, D. R. Riley, and J. J. Widrick, Physiology of a microgravity environment invited review: microgravity and skeletal muscle, J Appl Physiol. 89 (2000) 823-839.

[5] V. R. Edgerton, M. Y. Zhou, Y. Ohira, H. Klitgaard, B. Jiang, G. Bell, B. Harris, B. Saltin, P. D. Gollnick, R. R. Roy, and et al., Human fiber size and enzymatic properties after 5 and 11 days of spaceflight, J Appl Physiol. 78 (1995) 1733-1739.

[6] F. W. Booth, and C. R. Kirby, Changes in skeletal muscle gene expression consequent to altered weight bearing, Am J Physiol. 262 (1992) R329-332.

[7] D. B. Thomason, and F. W. Booth, Atrophy of the soleus muscle by hindlimb unweighting, J Appl Physiol. 68 (1990) 1-12.

[8] R. J. Talmadge, R. R. Roy, and V. R. Edgerton, Distribution of myosin heavy chain isoforms in non-weight-bearing rat soleus muscle fibers, J Appl Physiol. 81 (1996) 25402546.

[9] S. R. Price, Increased transcription of ubiquitin-proteasome system components: molecular responses associated with muscle atrophy, Int J Biochem Cell Biol. 35 (2003) 617628.

[10] D. J. Glass, Molecular mechanisms modulating muscle mass, Trends Mol Med. 9 (2003) 344-350. 
[11] N. Cros, J. Muller, S. Bouju, G. Pietu, C. Jacquet, J. J. Leger, J. F. Marini, and C. A.

Dechesne, Upregulation of M-creatine kinase and glyceraldehyde3-phosphate dehydrogenase: two markers of muscle disuse, Am J Physiol. 276 (1999) R308-316.

[12] N. Cros, A. V. Tkatchenko, D. F. Pisani, L. Leclerc, J. J. Leger, J. F. Marini, and C. A. Dechesne, Analysis of altered gene expression in rat soleus muscle atrophied by disuse, J Cell Biochem. 83 (2001) 508-519.

[13] S. C. Bodine, E. Latres, S. Baumhueter, V. K. Lai, L. Nunez, B. A. Clarke, W. T. Poueymirou, F. J. Panaro, E. Na, K. Dharmarajan, Z. Q. Pan, D. M. Valenzuela, T. M. DeChiara, T. N. Stitt, G. D. Yancopoulos, and D. J. Glass, Identification of ubiquitin ligases required for skeletal muscle atrophy, Science. 294 (2001) 1704-1708.

[14] J. St-Amand, K. Okamura, K. Matsumoto, S. Shimizu, and Y. Sogawa, Characterization of control and immobilized skeletal muscle: an overview from genetic engineering, Faseb J. 15 (2001) 684-692.

[15] M. Wittwer, M. Fluck, H. Hoppeler, S. Muller, D. Desplanches, and R. Billeter, Prolonged unloading of rat soleus muscle causes distinct adaptations of the gene profile, Faseb J. 16 (2002) 884-886.

[16] Y. W. Chen, G. A. Nader, K. R. Baar, M. J. Fedele, E. P. Hoffman, and K. A. Esser, Response of rat muscle to acute resistance exercise defined by transcriptional and translational profiling, J Physiol. 545 (2002) 27-41.

[17] T. Shoshani, A. Faerman, I. Mett, E. Zelin, T. Tenne, S. Gorodin, Y. Moshel, S. Elbaz, A. Budanov, A. Chajut, H. Kalinski, I. Kamer, A. Rozen, O. Mor, E. Keshet, D. Leshkowitz, P. Einat, R. Skaliter, and E. Feinstein, Identification of a novel hypoxia-inducible factor 1responsive gene, RTP801, involved in apoptosis, Mol Cell Biol. 22 (2002) 2283-2293. 
[18] D. F. Pisani, C. Cabane, B. Derijard, and C. A. Dechesne, The topoisomerase 1interacting protein BTBD1 is essential for muscle cell differentiation, Cell Death Differ. 11 (2004) 1157-1165.

[19] L. W. Ellisen, K. D. Ramsayer, C. M. Johannessen, A. Yang, H. Beppu, K. Minda, J. D. Oliner, F. McKeon, and D. A. Haber, REDD1, a developmentally regulated transcriptional target of p63 and p53, links p63 to regulation of reactive oxygen species, Mol Cell. 10 (2002) 995-1005.

[20] Z. Wang, M. H. Malone, M. J. Thomenius, F. Zhong, F. Xu, and C. W. Distelhorst, Dexamethasone-induced gene 2 (dig2) is a novel pro-survival stress gene induced rapidly by diverse apoptotic signals, J Biol Chem. 278 (2003) 27053-27058.

[21] L. Poellinger, and R. S. Johnson, HIF-1 and hypoxic response: the plot thickens, Curr Opin Genet Dev. 14 (2004) 81-85.

[22] G. L. Wang, B. H. Jiang, E. A. Rue, and G. L. Semenza, Hypoxia-inducible factor 1 is a basic-helix-loop-helix-PAS heterodimer regulated by cellular O2 tension, Proc Natl Acad Sci U S A. 92 (1995) 5510-5514.

[23] B. Jiang, Y. Ohira, R. R. Roy, Q. Nguyen, E. I. Ilyina-Kakueva, V. Oganov, and V. R. Edgerton, Adaptation of fibers in fast-twitch muscles of rats to spaceflight and hindlimb suspension, J Appl Physiol. 73 (1992) 58S-65S.

[24] Y. Ohira, W. Yasui, F. Kariya, T. Wakatsuki, K. Nakamura, T. Asakura, and V. R. Edgerton, Metabolic adaptation of skeletal muscles to gravitational unloading, Acta Astronaut. 33 (1994) 113-117.

[25] K. Almurshed, and K. Grunewald, The effects of dietary energy restriction on overloaded skeletal muscle in rats, Br J Nutr. 84 (2000) 697-704.

[26] K. S. Almurshed, and K. K. Grunewald, Dietary protein does not affect overloaded skeletal muscle in rats, J Nutr. 130 (2000) 1743-1748. 
[27] S. Schiaffino, and A. Serrano, Calcineurin signaling and neural control of skeletal muscle fiber type and size, Trends Pharmacol Sci. 23 (2002) 569-575. 


\section{Figure legends}

Figure 1. Multiple alignment of the SMHS1 protein sequence with vertebrate homologous protein sequences. Multalin (http://prodes.toulouse.inra.fr/multalin/multalin.html) and BoxShade (http://www.ch.embnet.org/software/BOX_form.html) programs were used. r: rat, m: mouse, $\mathrm{x}$ : xenopus, $\mathrm{z}$ : zebrafish. The respective GenBank accession numbers are:

\section{$\underline{\text { NP 536324 }}, \underline{\text { AAL39013 }}, \underline{\text { NP 989175 }}$ NP 543182, NP 956401.}

Figure 2. SMHS1 tissue expression analysis. A) Tissue distribution in rat. Multiple tissue Northern blot was hybridized with SMHS1 full-length cDNA probe. The 1,1 kb SMHS1 mRNA was detected in skeletal muscle, heart and testis. A human actin probe hybridizing to $\beta / \gamma \square(2 \mathrm{~kb})$ and $\alpha(1.6 \mathrm{~kb})$ actins according to tissue actin-isoform contents was used as control. B) Tissue distribution in mouse. Tissue RNA templates were used to detect SMHS1specific expression by RT-PCR. SMHS1 cDNA was amplified mainly from skeletal muscle and eye, and at a lower level from heart and brain. The 18S rRNA fragment was amplified as

control. C) SMHS1 in situ detection by RNA hybridization. Cryostat sections of 18 day-old mouse embryos were hybridized with an anti-sense or a sense SMHS1 riboprobe. SMHS1 was detected with the anti-sense riboprobe in limb muscles (sm), brain (b) and fetal heart (h). Original magnification, $2 \mathrm{x}(\mathrm{b}, \mathrm{sm}), 10 \mathrm{x}$ or $100 \mathrm{x}(\mathrm{h})$ and $200 \mathrm{x}(\mathrm{smf})$.

Figure 3. SMHS1 expression in differentiated $\mathrm{C}_{2} \mathrm{C}_{12}$ myotubes treated with $200 \mu \mathrm{M} \mathrm{CoCl}_{2}$. A) Total cell proteins were extracted and analyzed by Western blotting. HIF-1 $\alpha$ specific 120 $\mathrm{kDa}$ bands were immunodetected and $80 \mathrm{kDa}$ non-specific bands stained with amido black are shown as an even lane loading control. B) RNAs were prepared and analyzed by semiquantitative RT-PCR with specific primers to SMHS1 and to 18S rRNA. C) Band intensities 
were measured, and SMHS1 mRNA amounts are expressed as ratios between the different time- and the T0-band intensities after normalization by 18S rRNA variations. The results represent mean \pm S.E.M. of three independent RT-PCR experiments from two independent RNA preparations.

Figure 4. SMHS1 mRNA amounts and muscle mass variations. Soleus (A) and gastrocnemius (B) muscle atrophies were obtained from 14-day hindlimb suspended rats and rat soleus muscle compensatory hypertrophy (C) was obtained 6 weeks after gastrocnemius muscle ablations. Muscles were dried and weight just after excision. The muscle mass measurements represent mean \pm S.E.M. of 9-rat muscles of each groups. * : significantly different from control $(\mathrm{P}<0.003)$. Total RNAs were analyzed by Northern blot $(\mathrm{A}, \mathrm{C})$ or semiquantitative RT-PCR (B). Band intensities were measured and the results are expressed after normalization by $18 \mathrm{~S}$ rRNA variations. Results represent mean \pm S.E.M. of three independent RNA analyses from 9-rat soleus muscle pools. * : significantly different from control $(\mathrm{P}<0.001)$

Figure 5. Comparison of SMHS1 expression in predominantly oxidative soleus muscle (S) and predominantly glycolytic gastrocnemius muscle (Q). Total RNAs isolated from muscles excised from 9-rat pools were analyzed by semi-quantitative RT-PCR with SMHS1 or 18S rRNA specific primers. Band intensities were measured and the results are expressed in an arbitrary unit after normalization by $18 \mathrm{~S}$ rRNA variations. The results represent mean \pm S.E.M. of three independent RNA analysis from 9-rat soleus and gastrocnemius muscle pools. * : significantly different from soleus muscle $(\mathrm{P}<0.001)$. 
Figure 1.

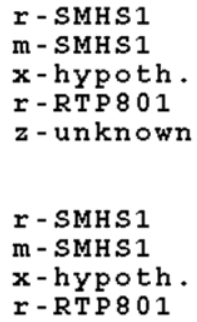


Figure 2.
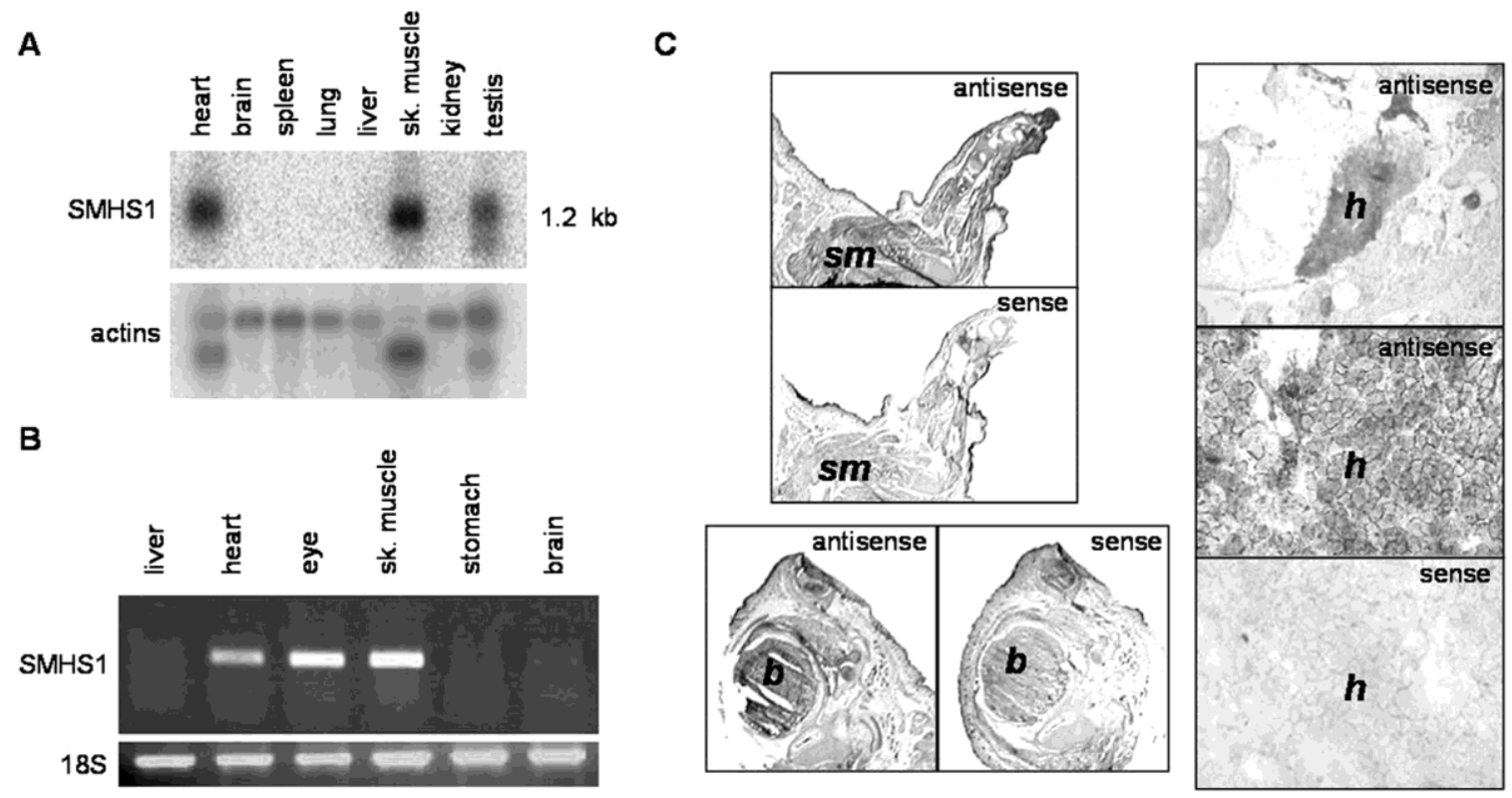
Figure 3.

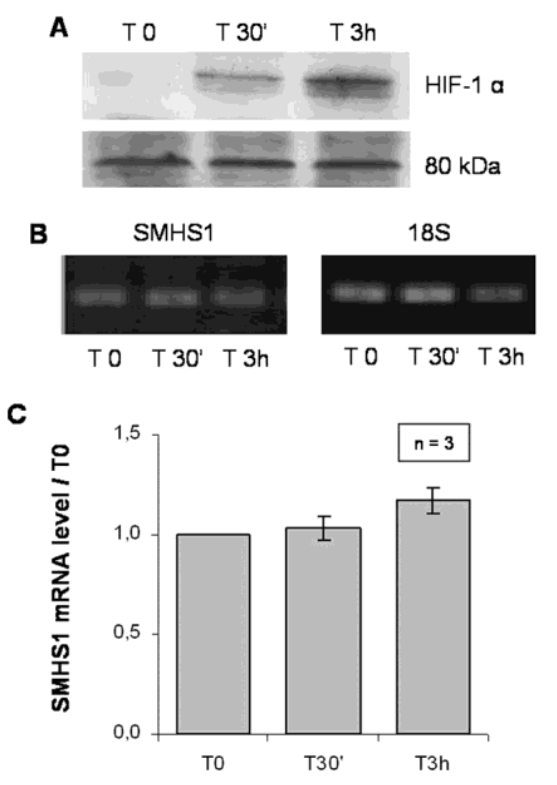


Figure 4.
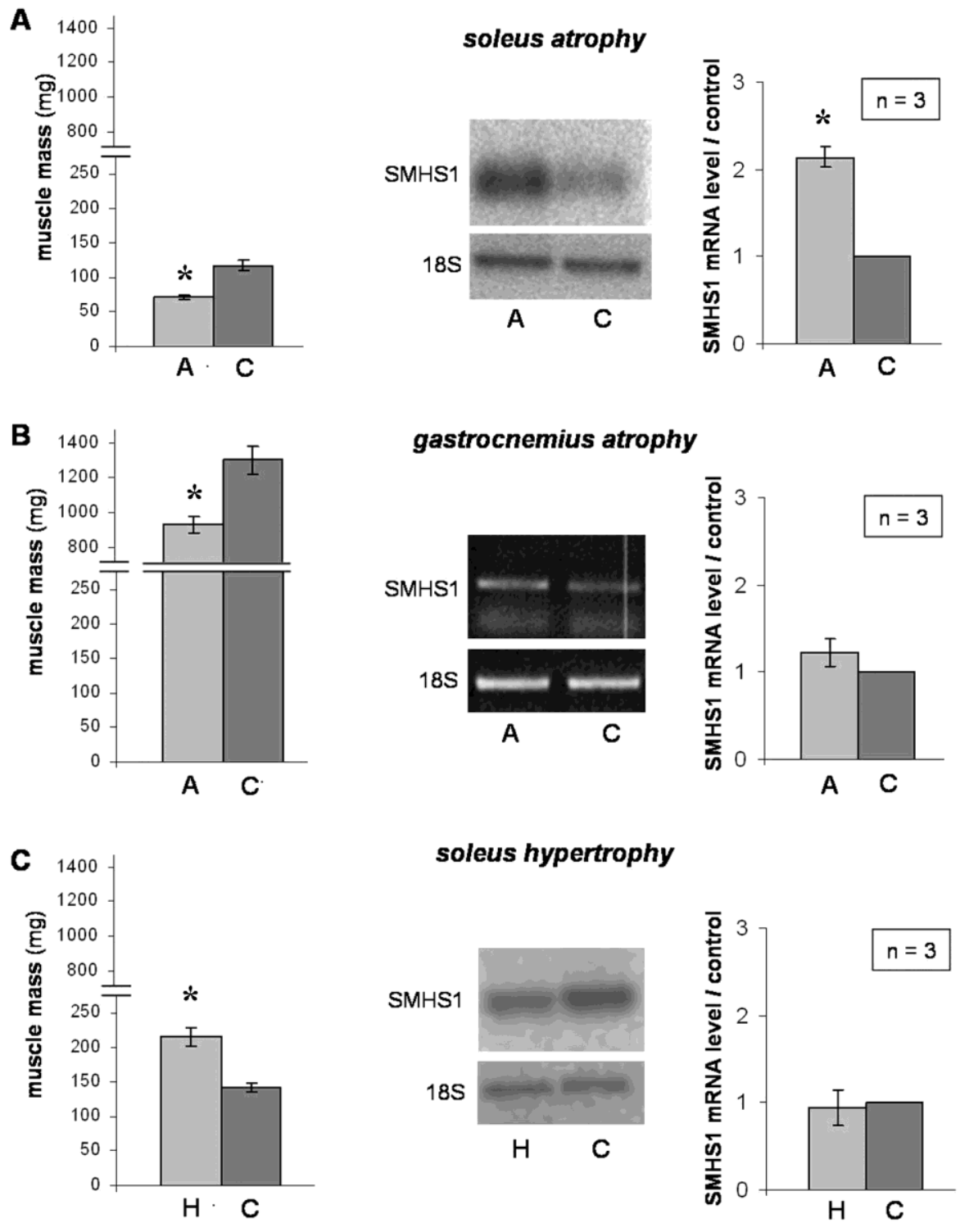

soleus hypertrophy

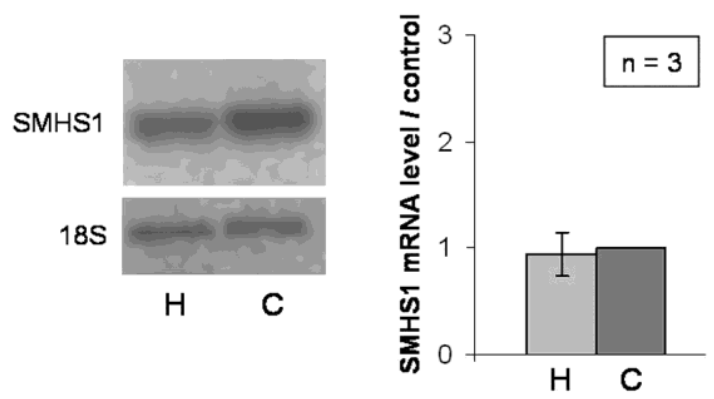


Figure 5.
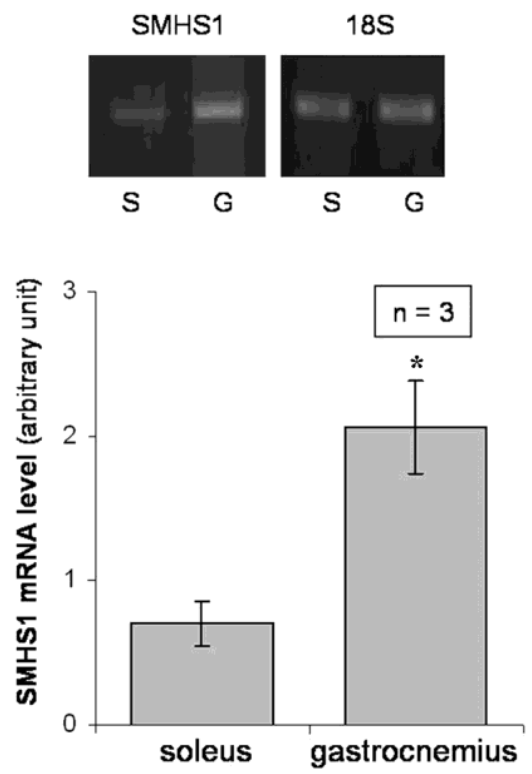